

\title{
Molecular Dynamics Studies of the Melting of Copper with Vacancies amd Dislocations at High Pressures
}

Clarence C Matthai and Jessica Rainbow

School of Physics and Astronomy, Cardiff University

Cardiff, UK.

\begin{abstract}
Molecular dynamics simulations of the melting process of bulk copper were performed using the Large-scale Atomic/Molecular Massively Parallel Simulator (LAMMPS) with the interatomic potentials being described by the embedded atom method. The aim of the study was to understand the effects of high pressures and defects on the melting temperature. The simulations were visualised using Visual Molecular Dynamics (VMD). The melting temperature of a perfect copper crystal, was found to be slightly higher than the experimentally observed value. The melting temperature as a function of pressure was determined and compared with experiment. Point and line defects, in the form of dislocations, were then introduced into crystal and the new melting temperature of the crystal determined. We find that the melting temperature decreases as the defect density is increased. Additionally, the slope of the melting temperature curve was found to decrease as the pressure was increased while the vacancy formation energy increases with pressure.
\end{abstract}

\section{INTRODUCTION}

The melting transition has been studied extensively over many decades and over the years many theories of this transition have been expounded. It had been suggested that melting occurs when the atom vibrations, as the crystal is heated, become large enough at the melting temperature such that the long range order is lost. Lindemann postulated that at the melting temperature could be approximately defined as the point at which the mean interatomic spacing exceeds its equilibrium spacing by $10 \%$. This is the so-called Lindemann criteria [1] for melting. It is now generally agreed that defects, and in particular line defects, play an important role in the melting transition. In dislocation theories of melting, the number of dislocations increases according to some power law at the transition temperature.

More recently, it has been suggested [2] that a wide variety of phase transitions may be formulated in terms of the formation of quasi-particles at the transition. In this phenomenological theory it was proposed that the transition temperature $T_{t}$ could be written in the form $k_{B} T_{t}=E_{\text {char }} e^{-\gamma}$ where $E_{\text {char }}$ is some characteristic energy associated with the transition and $k_{B}$ is the usual Boltzman constant. The quantity $\quad \gamma$ is related to the energy required to create the quasi-particle appropriate to the transition. It was further proposed that for melting the characteristic energy is related to the bulk modulus, $\mathrm{B}$, through the relation

$E_{\text {char }}=\Omega B$ where $\Omega$ is the atomic volume. The quasi-particles in this formulation are the phonons associated with the shear modulus. Point and line defects allow for the annihilation of these quasi-particles. In this study we have investigated the dependence of the melting temperature on the defect (point and line) density. In addition, we have also investigated the role 
of pressure, $p$, in melting as this could be used to test model theories. In particular, the slope of the calculated $T_{m}(p)$ curve is compared with experimental results.

\section{THEORY AND SIMULATIONS}

\section{The melting temperature as a function of pressure}

Simon and Glatzel [3] (SG) proposed an empirical relation between the melting temperature and the pressure, viz.,

$$
T_{m}(p)=T(0)\left[\frac{p}{a}+1\right]^{(1 / c)}
$$

where $a$ and $c$ are constants. This relationship only holds for situations where the melting temperature increases with pressure.

The slope of the melting curve at zero pressure has also been studied by many researchers. For example, Gilvarry constructed a theory in which the slope of the fusion curve at zero pressure could be expressed through the relation [4]

$$
\frac{1}{T_{m}(0)}\left[\frac{\partial T_{m}}{\partial p}\right]_{p=0}=(6 \gamma-2) \frac{1}{3 q_{0} B_{0}}
$$

In considering theories which relate the melting temperature to point and line defects, Matthai and March [5] used the results of a dislocation-mediated theory of the melting transition [6] to derive the equation

$$
\frac{1}{T_{m}(0)}\left[\frac{\partial T_{m}}{\partial p}\right]_{p=0}=\frac{1}{G} \frac{d G}{d p}
$$

They also used the empirical relation between the vacancy formation energy, $E_{v}^{f}$ and the melting temperature ( $E_{v}^{f} \sim 10 k_{B} T_{m}$ ) to give the gradient of the melting temperature curve at zero pressure in terms of the pressure dependence of the vacancy formation energy,

$$
\frac{1}{E_{v}^{f}(0)}\left[\frac{\partial E_{v}^{f}}{\partial p}\right]_{p=0}=\frac{1}{T_{m}(0)}\left[\frac{\partial T_{m}}{\partial p}\right]_{p=0}
$$

\section{$\underline{\text { Simulation Details }}$}

A 10x10x10 periodically repeating face centred cubic crystal with lattice spacing $3.615 \AA$ comprising 4000 atoms was constructed and the LAMMPS package [7] used to carry ou the molecular dynamics simulations. The Embedded Atom Method (EAM) interatomic potentials as formulated by Mei et al [8] which reproduced the equilibrium properties of $\mathrm{Cu}$ was used to characterise the interactions. The time step in the simulations were taken to be $1 \mathrm{fs}$ and the simulations were carried out at constant pressure using a NPT Nose-Hoover ensemble. In the pressure simulations, a hydrostatic pressure was applied to the simulation cell.

\section{Determination of the melting temperature}

In the molten state the long range order, present in the crystal, disappears and so the liquid state can be identified by examining the pair correlation function, which is in turn related to the structure factor. However, it is not feasible to determine $T_{m}$ from the structure factor 
data. In our simulations, the melting temperature was determined using three different approaches. In the first, the Lindeman criterion was applied by analysing the RMS displacement of the particles from their initial sites. The other two approaches involved plotting the volume per atom and the self-diffusion coefficient as a function of temperature. Both these latter quantities show a discontinuity at the melting temperature as can be seen in Figure 1. From all three approaches, the melting temperature of copper was found to be $1620 \mp 40 \mathrm{~K}$, which is much higher than the experimental value of $1354 \mp 5 \mathrm{~K}[9]$.
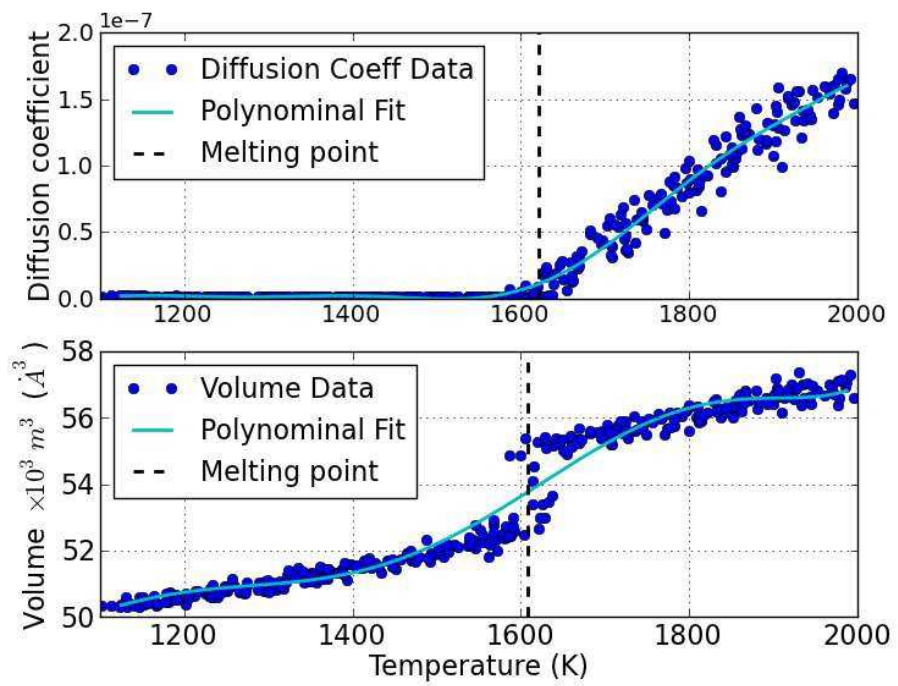

Figure 1: The diffusion coefficient shows a discontinuity in its slope at $T_{m}$, while the atomic volume is discontinuous at $T_{m}$.

\section{RESULTS}

\section{The melting temperature as a function of pressure}

The melting temperature as a function of pressure was determined by all the three approaches outline above. In the case of applying the Lindemann criterion, the pressure was limited to a maximum of $30 \mathrm{GPa}$. For the other two approaches the maximum pressure was extended to $300 \mathrm{GPa}$. The results for the $T_{m}(p)$, which are shown in Figure 2, are well fitted by the SG equation.Additionally, the results for the slope of the melting curve at zero pressure (Table 1) are in good agreement with the estimated value of $26.2 \mathrm{~K} \mathrm{GPa}^{-1}$ from the work of Japel et al [10].

\section{The effect of point defects on the melting temperature}

As seen above, the melting temperature for the perfect crystal was found to be much higher than the experimental value. In order to determine the effect of vacancies on the melting temperature, a number of vacancies were introduced into the crystal and simulations were repeated to determine the melting temperature. The results of these simulations are shown in Figure 3. 


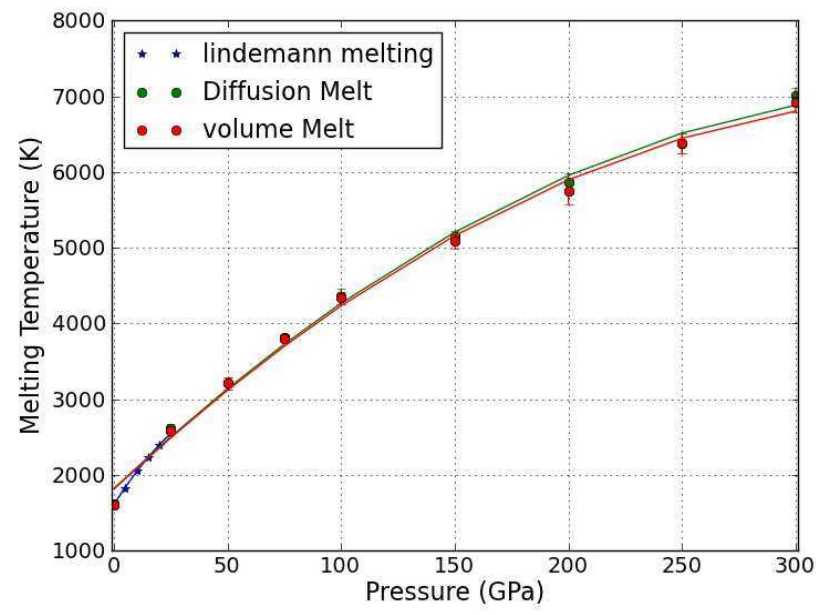

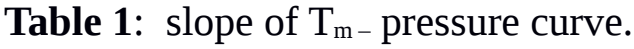

\begin{tabular}{|l|c|}
\hline Melting criteria & $\begin{array}{c}d T_{m} / d p \\
\left(\mathrm{~K} \mathrm{GPa}^{-1}\right)\end{array}$ \\
\hline Lindemann & 46.9 \\
\hline Diffusion & 28.3 \\
\hline Volume & 28.0 \\
\hline
\end{tabular}

Figure 2. The melting temperature as a function of pressure.

It is clear that as the number of vacancies is increased, $T_{m}$ decreases non-linearly until the vacancy number density is approximately $5 \%$ of the total number of $\mathrm{Cu}$ atoms. When the number of vacancies is increased further, $T_{m}$ is found to increase again. This partially due to the difficulty in identifying the melting temperature.

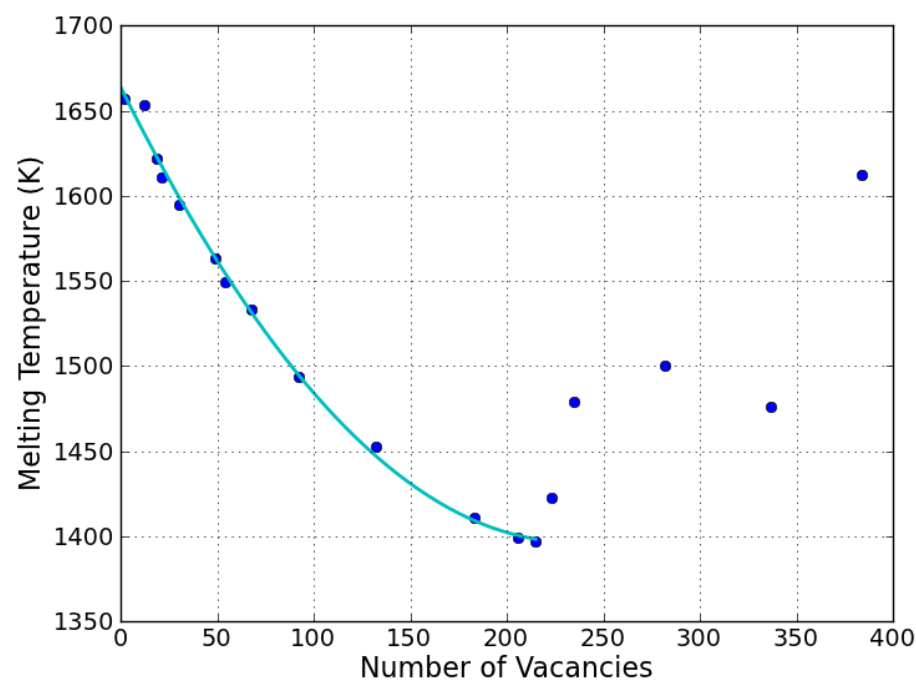

Figure 3. The melting temperature as a function of the number of vacancies.

We have also determined the pressure dependence of the mono-vacancy formation energy. This shows a power law increase over the pressure range investigated. One effect of this is to increase the melting temperature which is consistent with our simulations. Thus, it is clear the the mono-vacancy formation energy is intrinsically linked to the melting temperature.

\section{The effect of disclocations on the melting temperature}

It is generally agreed that while there is a relation between the vacancy formation energy and the melting temperature, it is more likely that line defects in the form of dislocations or 
disclinations are the drivers for the melting transition. We have therefore carried out simulations aimed at investigating how the dislocation density influences $T_{m}$.

Edge (see Figure 4) and screw dislocation loops of various lengths and different Burgers vectors were introduced into the crystal and the melting simulations carried out as before. The sites of the disclocation cores were randomly chosen. For the sake of consistency, the crystal block was taken to be the same size. This is not ideal as the number of atoms in the simulation box should ideally have been increased.
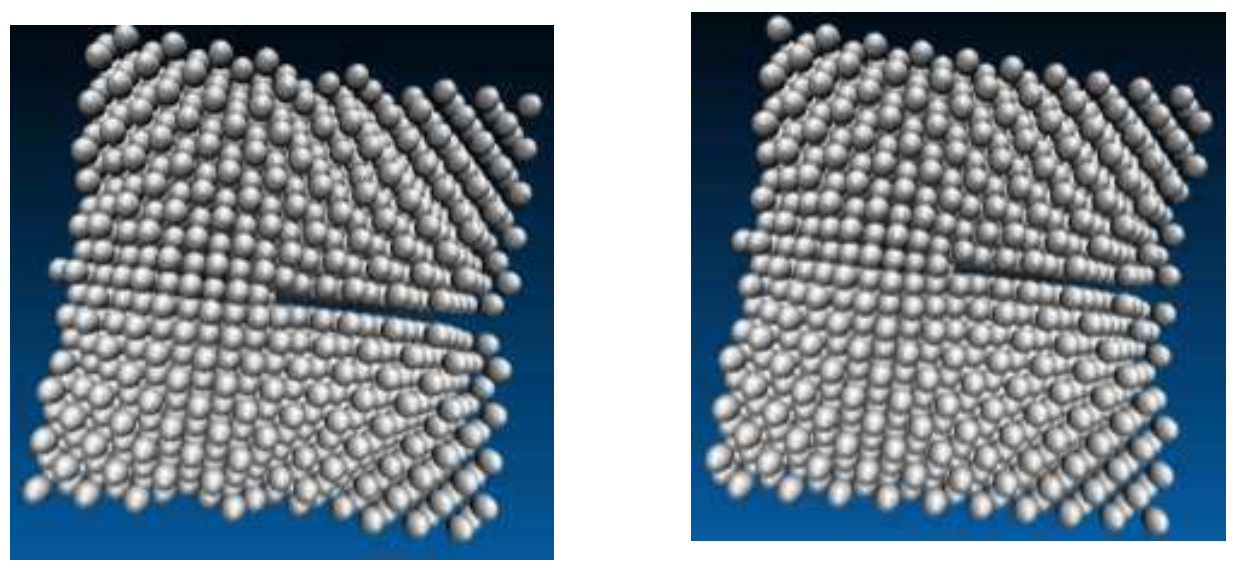

Figure 4. Structure of an edge dislocation before (left) and after (right) relaxation.

The melting temperature as a function of the number of dislocations is shown in Figure 5. As expected, the melting temperature does decrease with increasing dislocation density. Interestingly, the functional dependence appears to be linear in form.

\section{CONCLUSIONS AND DISCUSSION}

Computer simulations have been carried out to investigate the influence of point and line defects on the melting temperature. The melting temperature for the perfect crystal is found to follow the SG curve with coefficients $a \simeq 11.5 \mathrm{GPa}$ and $c \simeq 0.5$. However, the melting temperatures were found to be much higher than that measured experimentally. When defects in the form of vacancies or dislocations are introduced, the computed melting temperature is lowered. Thus, we have been able to demonstrate the necessity of including defects in order to obtain melting temperatures reasonably close to the experimentally measured values. The slope of the melting curve at zero pressure is a useful quantity that could be used to test the validity of models or simulation results. While the results of simulations on the defect-free crystal carried out in this work appear to agree with experiment, it is important that the simulations under pressure are also conducted on the crystal with defects. These investigations are currently under way. The functional form of the increase in the vacancy formation energy with pressure mirrors that of the melting energy curve which suggests that the results of simulations on crystals with defects will mirror that found for the defect-free crystal.

The main shortcoming of this work is in the use of a relatively small size crystal block. It is therefore important to demonstrate that the results are not dependent on crystal size by carrying out simulations on much larger crystal blocks. Also, the NPT ensemble as used in these 
simulations does not allow for the exchange of particles. It is also important to note that point defects might lead to substantial structural changes in the discloations similar to that reported by Frolov et al [11] in their work carried out on grain bourndaries.

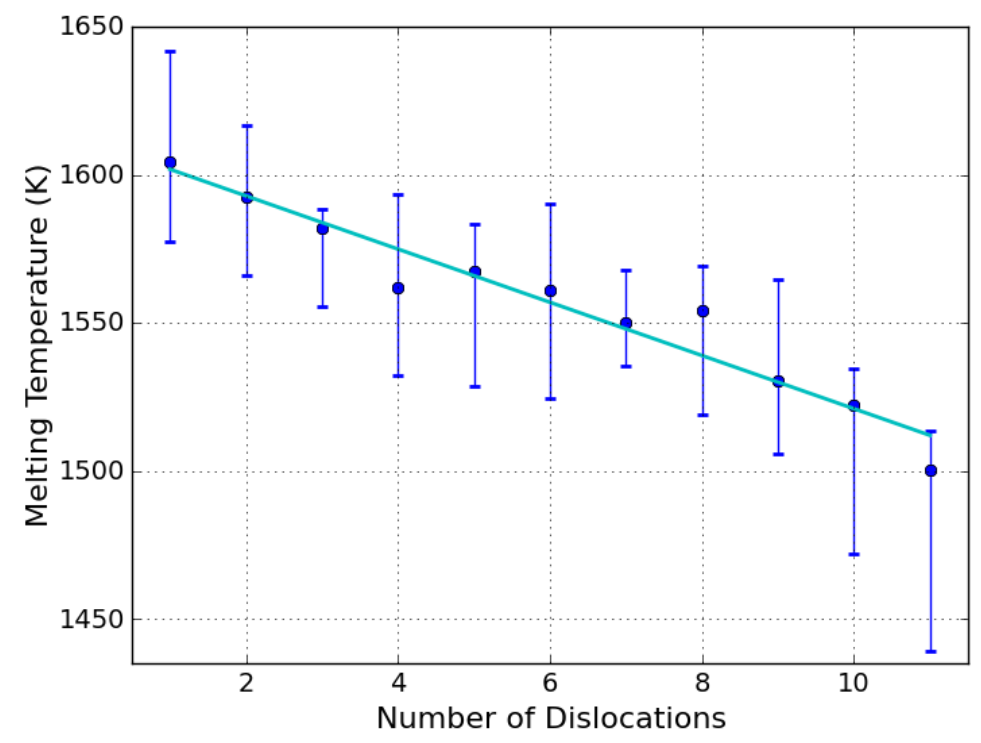

Figure 5. The variation of the melting temperature with increasing dislocation density.

\section{ACKNOWLEDGMENTS}

Some of the MD simulations were carried out on the ARCCA computing facilities at Cardiff University.

\section{REFERENCES}

1. F. A. Lindemann, Z. Phys. 11609 (1910).

2. N. H. March, E. V. Chulkov, P. M. Echenique and C. C. Matthai, Phase Transitions 831085 (2010)

3. F. E. Simon and G.Glatzel, Z. Anorg. Allg. Chem. 178309 (1929).

4. F. P. Bundy and H. M. Strong, in Solid State Physics 13, edited F. Seitz and D. Turnbull (Academic Press, New York, 1962), p. 81.

5. C.C. Matthai and N.H.March, Philos. Mag. Lett. 87475 (2007).

6. L. Burakovsky, D. L. Preston and R. R. Silbar, J. Appl. Phys. 886294 (2000).

7. LAMMPS Molecular Dynamics Simulator (2016). Available at: http://lammps.sandia.gov (accessed 19 April 2016).

8. J. Mei, J. W. Davenport, G. W. Fernardo, Phys. Rev. B: Condens.Matter, 434653 (1991).

9. H. Brand, D. P. Dobson, L. Vocaldo and I. G. Wood, High Press. Res. 26185 (2006).

10. S. Japel, B. Schwager, R. Boehler and M. Ross, Phys. Rev. Lett. 95167801 (2005).

11. T. Frolov, D. L. Olmstead, M. Asta and Y. Mishin, Nat. Commun. 41899 (2013).

T. Frolov, M Asta and Y. Mishin, Phys Rev B 92020103 (2015).

S. von Alfthan, P. D. Haynes, K. Kaski and A. P. Sutton, Phys. Rev. Lett. 96, 055505 (2006). 